\title{
Quantum Fluctuations of a Single Trapped Atom: Transient Rabi Oscillations and Magnetic Bistability
}

\author{
H. Schadwinkel, V. Gomer, U. Reiter, B. Ueberholz, and D. Meschede \\ Institut für Angewandte Physik, Universität Bonn, \\ Wegelerstr. 8, D-53115 Bonn, Germany
}

November 1, 2018

\begin{abstract}
Isolation of a single atomic particle and monitoring its resonance fluorescence is a powerful tool for studies of quantum effects in radiationmatter interaction. Here we present observations of quantum dynamics of an isolated neutral atom stored in a magneto-optical trap. By means of photon correlations in the atom's resonance fluorescence we demonstrate the well-known phenomenon of photon antibunching which corresponds to transient Rabi oscillations in the atom. Through polarization-sensitive photon correlations we show a novel example of resolved quantum fluctuations: spontaneous magnetic orientation of an atom. These effects can only be observed with a single atom.
\end{abstract}

keywords: laser cooling and trapping, single atoms, photon correlations, quantum fluctuations

\section{Introduction}

Radiation matter interaction has been studied for a long time with atomic samples. It is known that density matrix theory is well suited to exhaustively describe the properties of fluorescence from macroscopic atomic ensembles, and that in this limit semiclassical theory gives excellent approximations, since phenomena related to the quantum nature of the light field are often hidden.

On the other hand, it has also been realized since more than two decades now that isolation of a single atom provides an opportunity to directly observe pure quantum properties of the interacting radiation-matter system. A celebrated example is the observation of photon antibunching, where initial experiments [1], 2] were carried out with extremely diluted atomic beams, 
and more precise investigations became possible when ion traps were employed to achieve long-term confinement of a single atomic particle [3].

For neutral atoms the confinement strength is reduced since trapping has to rely on forces derived from electric or magnetic dipole interaction. Therefore neutral atoms have only more recently become available for measurements at the microscopic level through the application of laser cooling techniques well established for macroscopic samples with many atoms. With laser light single neutral atoms can be trapped and observed for long times [4, 司] in analogy with ion traps.

One of the most powerful methods to observe quantum fluctuations in atom-radiation interaction is to measure photon correlations of the light emitted by a single atom. The quantum theoretical description of photodetection was put forward by Glauber [6] in the early 60s, and very successfully applied to all appropriate experiments since. In the photon language the normalized intensity-intensity correlation function is the conditional probability to detect a second photon if a first one was detected a time $\tau$ before. This second order correlation function is defined by

$$
g^{(2)}(\tau)=\frac{\langle: \hat{n}(t+\tau) \hat{n}(t):\rangle}{\langle\hat{n}(t)\rangle\langle\hat{n}(t)\rangle},
$$

where $\hat{n}(t)=\hat{a}^{\dagger}(t) \hat{a}(t)$ is the photon number operator constructed from field operators $\hat{a}^{\dagger}, \hat{a}$, and where $: \hat{n}(t+\tau) \hat{n}(t)$ : denotes normal ordering of field operators. At sufficiently long delays $\tau$ all possible correlations have decayed, and hence $\langle: \hat{n}(t+\tau) \hat{n}(t):\rangle \rightarrow\langle\hat{n}(t)\rangle\langle\hat{n}(t)\rangle$. It is thus often informative to discuss the deviation of $g^{(2)}(\tau)$ from unity, or the quantity $\left|g^{(2)}(\tau)-1\right|$ as a measure of the fluctuation strength of the system, called contrast in the following.

For auto correlations (11) all classical fields must obey $g^{(2)}(0) \geq 1$, while photon antibunching shows $g^{(2)}(0)=0$, making it a prime example of quantum field fluctuations. In an intuitive interpretation it is said [7 that the first detected photon projects an atom in its ground state. From this initial state the atom then relaxes back to its equilibrium state on the time scale of its radiative decay. In this sense the observation of this fluctuation is 'measurement induced'.

In our work we are additionally interested in the case where different atomic transitions can be distinguished by their polarization properties. We therefore analyze cross correlations of orthogonal polarization states $|\beta\rangle$ and $|\alpha\rangle$ of the resonance fluorescence from a single atom,

$$
g_{\alpha \beta}^{(2)}(\tau)=\frac{\left\langle: \hat{n}_{\alpha}(t+\tau) \hat{n}_{\beta}(t):\right\rangle}{\left\langle\hat{n}_{\alpha}(t)\right\rangle\left\langle\hat{n}_{\beta}(t)\right\rangle} .
$$

A single trapped Cesium atom shows very strong polarization correlations in its resonance fluorescence. While this effect is very pronounced for correlations between orthogonal circular polarizations of the detected photons, 
it vanishes for linearly polarized photons even in a light field with linear polarization at every place. It is the purpose of this work to show that the strong contrast in $g_{\alpha \beta}^{(2)}(\tau)$ that we observe for orthogonally circular polarization states in the fluorescence of a single trapped atom can be interpreted as a direct consequence of the atomic orientation or magnetization undergoing spontaneous or quantum fluctuations.

\section{Photon antibunching and transient Rabi oscil- lations}

All classical fields have auto correlations $g^{(2)}(0) \geq g^{(2)}(\tau)$, and a value $g^{(2)}(0)-1<0$ is classically forbidden (for classical fields one should replace all operators $\hat{n}(t)$ in (1) by their classical counterparts, intensities $I(t))$. This enhanced probability to detect two photons simultaneously is called 'photon bunching' and was observed as early as in 1956 [8] from a usual thermal light source.

For a single-atom fluorescence, however, $g^{(2)}(\tau)$ vanishes identically for $\tau=0$ which is a reflection of the fact that one can find at most one photon in the field mode of interest and can never detect two photons simultaneously. This phenomenon is called 'photon antibunching' and is regarded as an important manifestation of the quantum nature of light.

Since the emitted light field reflects the evolution of the atomic dipole moment the correlation function $g^{(2)}(\tau)$ visualizes the internal dynamics of the observed atom for $\tau>0$. The state of an excited atom evolves continuously in the absence of a measurement, but theory predicts a sudden projection to the ground state when a photon is detected.

This measurement 'triggers' the atom to the initial conditions $\rho_{g g}(0)=1$ and $\rho_{e e}(0)=0$, where $\rho_{g g}(t)$ and $\rho_{e e}(t)$ represent the population of the ground and excited atomic state, respectively. At that instant the coherent evolution starts again from the values $\rho_{g g}(0)=1$ and $\rho_{e e}(0)=0$ and will be interrupted by the next spontaneous emission. The normalized probability for detecting a second spontaneously emitted photon is now proportional to the population of the excited atomic state $\rho_{e e}$ according to [9]

$$
g^{(2)}(\tau)=\rho_{e e}(\tau) / \rho_{e e}(\infty) .
$$

Since the emission times are random, after averaging over many evolution trajectories the measured $g^{(2)}(\tau)$ shows directly relaxation of the system back to the equilibrium state after its wave function has collapsed due to the first photon detection.

In fig. 1 we show $g^{(2)}(\tau)$ of resonance fluorescence from a single atom stored in a magneto-optical trap (MOT) [10] with clearly observable photon antibunching and transient oscillations. As we already reported previously 


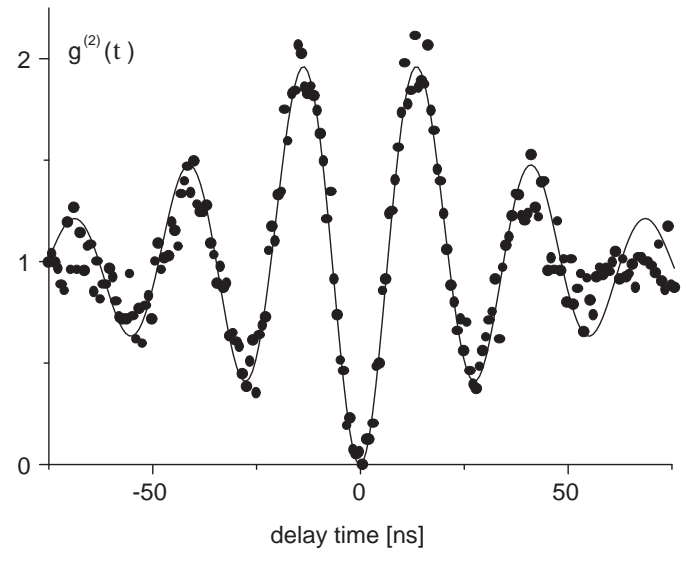

Figure 1: Intensity-intensity correlations in the resonance fluorescence of a single Cs atom stored in a MOT. The uncorrelated background of straylight photons has been measured independently and subtracted. Details can be found in Ref. [4, 河.

[4], the observed transient oscillations in the population of the excited state in Fig. 1 corresponding to coherent excitation and deexcitation cycles (Rabi oscillations) can be surprisingly well described by a simple model (solid line in Fig. 1) of a two-level atom (similar to $F=0 \rightarrow F=1$ transition) in spite of the complicated multilevel structure of the Cesium atom and light interference pattern (see below). This observation suggests that due to optical pumping a trapped atom spends most of its time in the magnetic substate that interacts most strongly with the local field and is forced to behave, to a good approximation, like a two-level system. For $\tau$ larger than the life time of the excited state the correlations die out due to the fluctuations of the vacuum field.

\section{Orientation dynamics of the atom revealed by polarization correlations}

An atom trapped in a MOT and moving through the light interference pattern experiences various intensities and polarizations at different places. The polarization of the resonance fluorescence is determined by the atomic interaction with the local light field and changes on the time scale of atomic transport over an optical wavelength $\lambda$. Thus, in addition to correlations of the total intensity one expects also polarization effects, that is correlations $g_{\alpha \beta}^{(2)}(\tau)$ measured between any polarization components $\alpha$ and $\beta$ which should strongly depend on the atomic motion and the light-field topography. 
The light field of the MOT is formed by three mutually orthogonal pairs of counterpropagating laser beams with $\sigma^{+}$and $\sigma^{-}$polarization. A pair of two circularly polarized laser beams with the same handedness produces a local polarization that is linear everywhere with a direction of polarization that rotates a full turn every half wavelength. With two additional 'polarization screws' for other directions along with the relative phases $\phi$ and $\psi$ between these standing waves one obtains for the total electric field in a $3 \mathrm{D}$ $\mathrm{MOT}_{\phi \psi}$

$\vec{E}=\left(\sin k z+\sin k y \cdot e^{i \psi}\right) \hat{x}+\left(\cos k z+\cos k x \cdot e^{i \phi}\right) \hat{y}+\left(\sin k x \cdot e^{i \phi}+\cos k y \cdot e^{i \psi}\right) \hat{z}$.

We have already reported on strong correlations between circularly polarized photons and vanishing correlations between linear polarization components observed in the resonance fluorescence of a single atom trapped in a standard MOT light field configuration [4]. This result seems to be intuitive only for specific choices of time phases: for example, $\phi=90^{\circ}, \psi=0$ yields an 'antiferromagnetic' light-field structure with alternating right- and left-hand circular polarizations at points of deepest light shift potential, see [11. However, in a standard MOT the phases $\phi$ and $\psi$ change randomly due to acoustic jitter and thermal drifts. Thus the trapping light field has no well defined polarization state and the correlations are averaged over all possible values of $\phi$ and $\psi$.

Since the MOT light field topography (4) strongly depends on the relative time-phases of the three contributing standing waves, we have chosen a setup where $\phi$ and $\psi$ are intrinsically stable [12]. The concept uses a single standing wave which is multiply folded and brought into triple intersection with itself. The phases $\phi$ and $\psi$ can be adjusted by means of Faraday rotators. Details of this approach have been published elsewhere [12, 11].

If one models an atom by a "classical emitter" $(F=0 \rightarrow F=1$ transition or steady-state density matrix of a multi-level atom) then its induced dipole moment will be proportional to the local light field. This idea is often used for interpretation of polarization correlations in the fluorescence of a large number of laser-cooled atoms [13]. As we will show this description fails completely in the case of a single atom.

The most interesting case occurs for $\phi=\psi=0$. In this situation the three standing waves oscillate synchronously and thus the interference light field has a linear polarization at every point and lacks handednees completely. In this case the model of a classical emitter [5] predicts strong correlations between orthogonal linear polarization components and relatively weak correlations between circular components in clear contradiction with experimental results (see below). 


\section{Experimental Setup}

For polarization-sensitive correlation measurements we have trapped individual neutral atoms in a standard six-beam MOT [10] with the only exception that the phases and hence the light field topography is fully controlled [12, 11]. At a quadrupole field gradient of $12.5 \mathrm{G} / \mathrm{cm}$ the storage volume extends over approximately $100 \mu \mathrm{m}$. In order to trap small, countable numbers of atoms the loading rate from the background atomic vapour into the trap is kept very low. This is achieved on the one hand by lowering the Cesium partial pressure to $\approx 10^{-15}$ mbar (at a base pressure of $5 \cdot 10^{-10} \mathrm{mbar}$ ) and on the other hand by using trapping laser beams of diameter $4 \mathrm{~mm}$ only. The average number $\langle N\rangle$ of trapped atoms (typically between 1 and 5 in this experiment) can be easily adjusted by variation of the Cesium pressure. Although $\langle N\rangle$ also depends on the trapping laser intensity $I$ and detuning $\delta$ of the trapping laser from atomic resonance, we are able to observe trapping of individual atoms over a wide range of parameters: $0.3 I_{0}<I<3.6 I_{0}$ per laser beam and $-5.2 \Gamma<\delta<-1.1 \Gamma$. The natural linewidth and the saturation intensity of the cooling transition are $\Gamma=2 \pi \times 5.2 \mathrm{MHz}$ and $I_{0}=1.1$ $\mathrm{mW} / \mathrm{cm}^{2}$, respectively.

The atomic resonance fluorescence is due to excitation by the trapping laser field only. Fluorescent light is collected from a 5\% solid angle by a lens and then splitted into orthogonal polarization states by means of polarizing optics (see Fig. 2) which also directs the corresponding light onto two avalanche photo diodes (APD). The APDs are operated in single photon counting mode and achieve a photon detection efficiency of $47 \%$ at a dark count rate of $10 \mathrm{~s}^{-1}$. The average photon count rate for an individual atom lies in the range $3-10 \mathrm{kHz}$ in our experiments, depending on laser intensity and detuning. Observation direction is in the xy-plane at $45^{\circ}$ to the laser beams (the $z$-axis is the symmetry axis of the MOT quadrupole magnetic field).

Usually, a measurement of the cross correlation function along with the total intensity-intensity correlation provides complete information: the corresponding auto correlation can be inferred from the sum rules for orthogonal polarization components [5]. For example for circular components one

has $g_{++}^{(2)}(\tau)+g_{+-}^{(2)}(\tau)=2 g^{(2)}(\tau)$. A cross correlation measurement $(+-)$ can be carried out 4 times faster than the corresponding auto correlation measurement $(++)$ where only one half of the total fluorescence is detected.

A computer registers the arrival times of all photons from the two APD channels with $100 \mathrm{~ns}$ time resolution and with $700 \mathrm{~ns}$ dead time in each channel. The entire experimental information accessible in the set-up is stored and thus can be processed afterwards by correlation analysis through numerical multi-stop procedures, which completely eliminate systematic errors such as photon pile-up introduced by single-stop methods traditionally used in Hanbury Brown \& Twiss type experiments [8, 14]. 


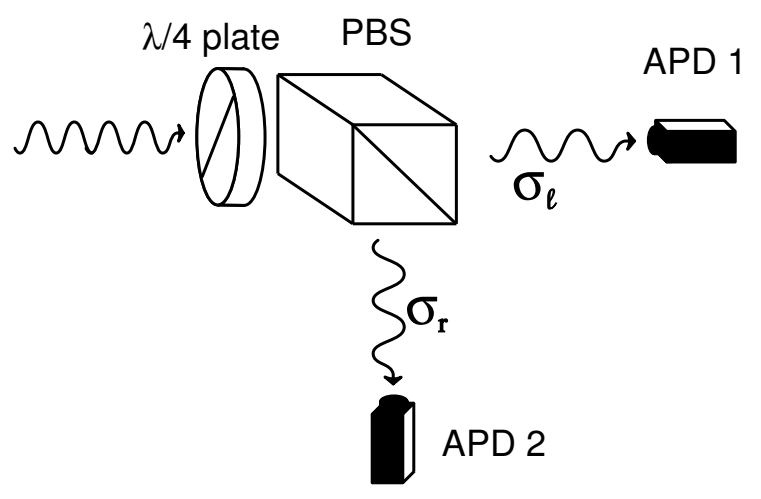

Figure 2: Optics for polarization-resolved photon correlations. To measure correlations between right and left handed $\ell / r$, ("circular correlations") a quarter wave plate is placed before the polarizing beam splitter (PBS). For vertical and horizontal $v / h$ ("linear correlations") components only a polarizing beam splitter is used. For total intensity-intensity correlations the fluorescence is divided by a non-polarizing beam splitter.

Atoms are randomly loaded from background vapour and randomly lost due to collisions with background gas. But since individual atom arrival and departure events are easily located within $1 \mathrm{~ms}$, it is straightforward to determine the instantaneous number of atoms from the average count rate. Note that the number of trapped atoms fluctuates on the second time scale [5]. This enables us to separate all data from a single experimental run into different classes with the number of trapped atoms as a parameter - the data for different atom numbers are therefore obtained under identical experimental conditions. It is also easily possible to distinguish correlations of the fluorescence of trapped individual atoms from uncorrelated background of detection events due to stray light or fluorescence from thermal, untrapped atoms. As a consequence all measured correlation functions can always be unequivocal normalized.

\section{$5 \quad$ Experimental Results}

Measured second order correlation functions for orthogonal polarization components in the fluorescence of a single atom in the $\mathrm{MOT}_{00}$ are shown in fig. 3. Within our experimental uncertainties correlations are completely absent for linear polarization components of the fluorescence, in baffling contrast with the result for circularly polarized components.

For a single atom the circular correlation contrast reaches s values up to $62 \%$. This very strong correlation typically relaxes within a few $\mu$ s. Since photon antibunching and Rabi oscillations at ns time scales are not resolved in these measurements, we have assumed for a simple analysis the relaxation 

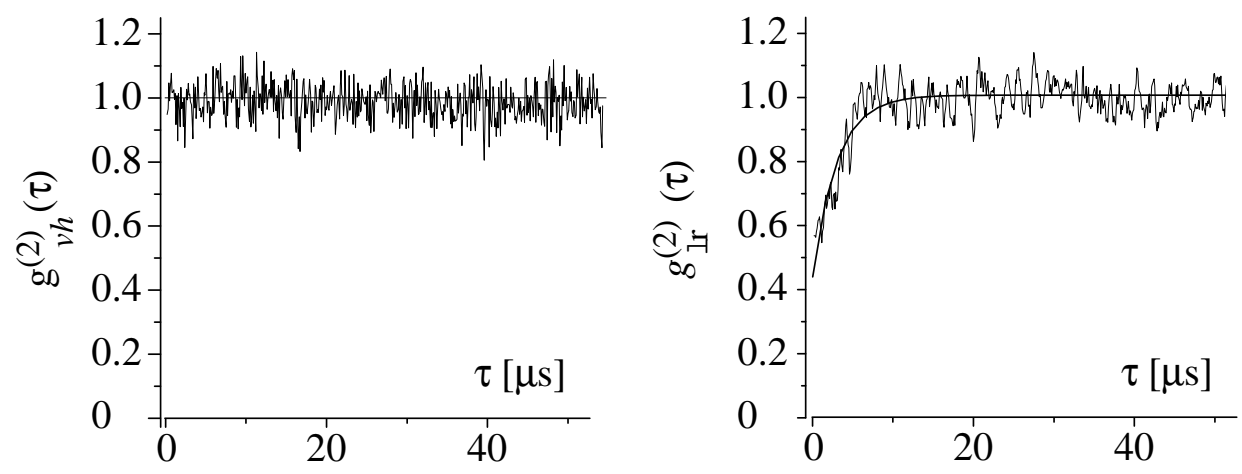

Figure 3: Measured photon correlations from a single atom in the $M O T_{00}$ field. Left: Correlation function $g_{v h}^{(2)}(\tau)$ for orthogonal linear polarization components $\left(\delta=-1.8 \Gamma, I=0.95 I_{0}\right.$, integration time 6.3 min). Right: Correlation function $g_{\ell r}^{(2)}(\tau)$ for orthogonal circular polarization components $\left(\delta=-2.7 \Gamma, I=0.7 I_{0}\right.$, integration time $\left.11.1 \mathrm{~min}\right)$. Solid line: exponential fit.

process of the polarization correlations to be exponential and extracted a single relaxation time constant $\tau_{r}$. The intensity-intensity auto correlation $g^{(2)}(\tau)$ also shows a contrast of about $30 \%$ due to intensity modulations of the MOT light field, but the relaxation time constant of about $0.6 \mu \mathrm{s}$ in fig. 4 is significantly shorter than for the circular cross correlations [15].

Furthermore, we have experimentally verified that $\tau_{r}$ does not depend on the number of trapped atoms and that the contrast of the correlation function is proportional to the inverse number of atoms, $N^{-1}$ (see fig. 5). Thus, as one would expect, we deal with a pure single-atom effect.

\section{Discussion}

In order to interpret the polarization properties of the resonance fluorescence of atoms driven by a light field with linear polarization we begin by considering an atom at rest. As this light field does not favor any of the two circular polarization states, one can assume that prior to the detection of the first photon the distribution of atomic magnetic sublevels is symmetric, $\langle m\rangle=0$. If we suppose for simplicity that the local light field consists of equal parts of both orthogonal circular polarizations only, it is clear that the $\langle m\rangle=0$ state is unstable [16]. At the level of an individual particle this equilibrium state can be distorted by the observation of a single circularly polarized photon, which projects the atom into its ground state, breaks the symmetry of the Zeeman substate population (fig. 6), and creates an imbalance in the interaction strengths with both circular polarization components. The next absorption will be preferentially further enhance the asymmetry. 


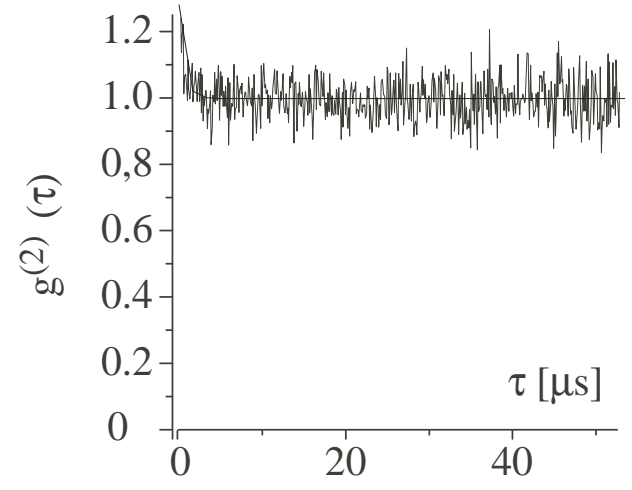

Figure 4: Total intensity-intensity correlation function $g^{(2)}(\tau)$ of a singleatom fluorescence in the $M O T_{00}$ field $\left(\delta=-1.1 \Gamma, I=1.3 I_{0}\right.$, integration time $10.4 \mathrm{~min})$. Solid line: exponential fit.
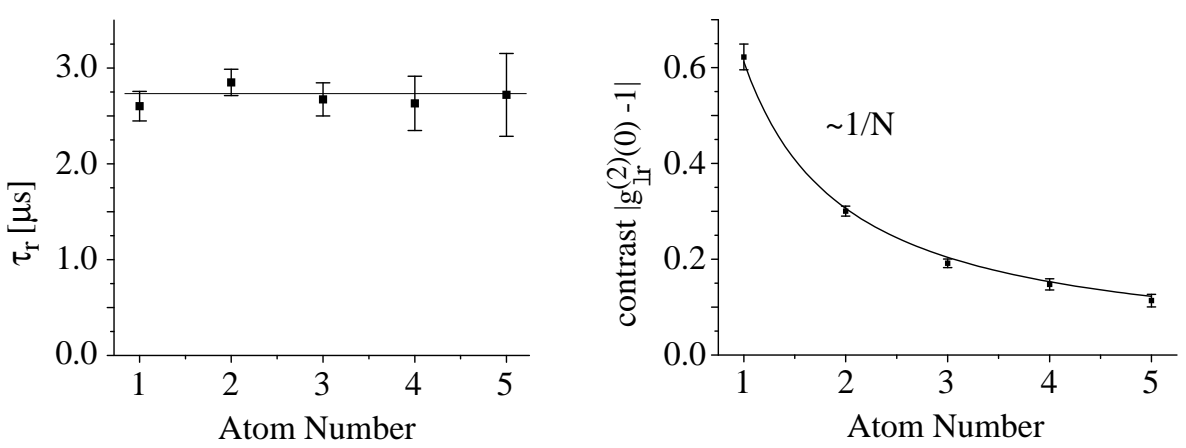

Figure 5: Measured characteristic relaxation time (left) and contrast (right) of the cross correlations for circular polarization components as a function of the number of observed atoms recorded at constant trap parameters.

The imbalance in the interaction strengths rapidly grows with $m$ leading to fast pumping into one of the outmost Zeeman states $m= \pm F$. The ratio of the interaction strengths in these stretched states reaches the value $(2 F+1)(F+1)$ making them very stable for large $F$ even in the presence of the polarization component in the light field driving $\Delta m=0$ transitions. The atom in this oriented state prefers to radiate into the same polarization state as the first detected photon, resulting in anticoincidences in the cross correlation for orthogonal circular polarizations.

Note that this effect is a specific feature of $F \rightarrow F+1$ transitions and does not occur for $F \rightarrow F$ or $F \rightarrow F-1$ transitions. It can be regarded as bistability, since an emission of a circularly polarized photon makes the atom more likely to be pumped in the corresponding outmost Zeeman state. As both stretched states $m=+F$ and $m=-F$ are equivalent in the presence of a linear polarized light field, this effect is principally unobservable 
in an atomic ensemble. However, if one adds some circular polarization component to the driving light field a spontaneous spin polarization of a macroscopic sample can be observed as recently demonstrated in 16 by optically pumping on the $F=3 \rightarrow F=4$ hyperfine component of the $D_{1}$-line of Cs.

Although the real 3D-situation in our experiment is much more complicated than the simple model presented above, our interpretation is furthermore supported by the following considerations: As noted in [16], the condition for magnetic bistability in a linearly polarized light field is given by $\left|90^{\circ}-\beta\right|<45^{\circ}$, where $\beta$ is the angle between the magnetic field (quantization axis) and the light polarization. In the $\mathrm{MOT}_{00}$ there are 8 intensity antinodes in a unit cell with directions of the local linear polarization at these points coinciding with the diagonals of the coordinate system $( \pm 1, \pm 1, \pm 1)$. It is easy to see that the bistability condition is fulfilled for the majority of points in the MOT magnetic quadrupole field $B \propto(-x,-y, 2 z)$.

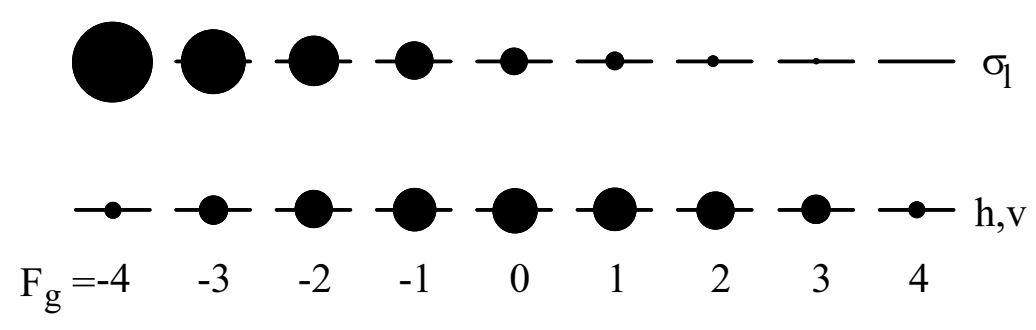

Figure 6: Ground state population after decay of an initially isotropic exited state distribution with $\langle m\rangle=0$ by emitting a circular polarized photon $\sigma_{l}$ (upper part). The dot sizes correspond to the individual populations of the sublevels. For comparison, the situation is completely different for the hvcorrelation: Detecting a linearly $h$ - or v-polarized photon (lower part) does not lead to any orientation of the atom. Therefore any succeeding excitation will result in almost equal probabilities for detecting a $h$-or a v-polarized photon in the next spontaneous emission, leading to $g_{h v}^{(2)}(\tau=0) \approx 1$.

However, in our case the stretched states are not intrinsically stable. While radiation pressure forces are balanced for an aligned atom with $\langle m\rangle=0$, they are unbalanced for an oriented atom since the local linearly polarized light field is created by (at least two) counterpropagating laser beams with orthogonal circular polarizations. The imbalance in the light forces created by atomic orientation thus causes acceleration, or heating which is again damped by the usual laser friction forces 17]. Thus for our experiment we must acknowledge that the observation of a circularly polarized photon not only redefines atomic orientation but also its mechanical status: Internal and external atomic degrees of freedom are inextricably entangled.

The measured relaxation time constant of $g_{\ell r}^{(2)}(\tau)$ indeed depends strongly on the atom-light field interaction which also governs atomic motion in the 
trap. The interaction strength is measured by the light shift parameter $\Lambda$ [18, 11] corresponding to the maximum energy shift of the atomic energy levels. Under our experimental parameters sub-Doppler cooling leads to atomic temperatures proportional to the light shift, thus $T_{k i n} \propto \Lambda$ [10, 17, 19].

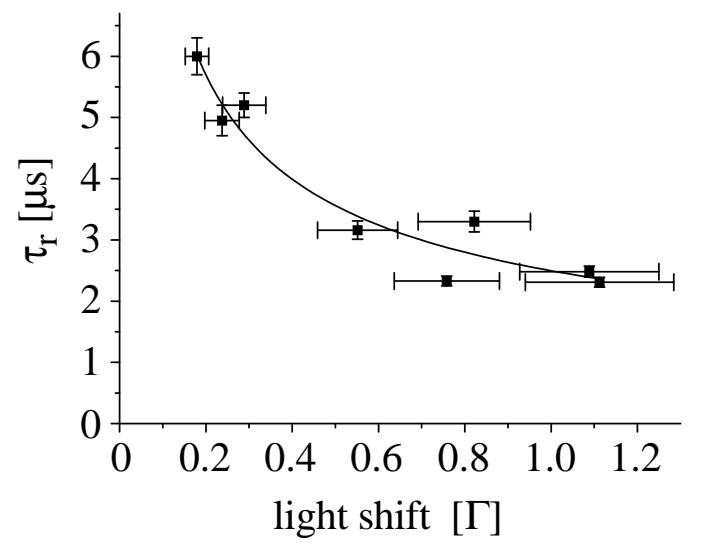

Figure 7: The relaxation time constant $\tau_{r}$ as a function of the light shift parameter $\Lambda$. Solid line: fit function $\propto \Lambda^{\alpha}$, with $\alpha=-0.51 \pm 0.01$.

In fig. 7 we show the measured dependence of the time constant $\tau_{r}$ as a function of $\Lambda$. As expected we find good agreement of our experimental data with the functional relationship $\tau_{r} \propto 1 / \bar{v} \propto 1 / \sqrt{T_{k i n}} \propto 1 / \sqrt{\Lambda}$. Here $\bar{v}$ denotes the average atomic velocity. This means that relaxation of the spontaneous atomic magnetization is determined by atomic motion through the light field.

We can carry out our analysis one step further if we assume that the characteristic length over which relaxation takes place is $\tau_{r} \bar{v}=\lambda / 2$, the spatial period of the MOT light field. It is then straightforward to evaluate characteristic kinetic temperatures from our correlation measurements to be in the range between $10-68 \mu \mathrm{K}$ for the $\Lambda$ range shown in fig. 7 , in good agreement with previous measurements [10, 19].

\section{Summary}

Photon correlations observed in the resonance fluorescence of a single atom provide a direct access to the internal atomic dynamics. We have shown two examples of resolved quantum dynamics of an isolated atom stored in a magneto-optical trap. Beyond observation of the well-known phenomenon of transient Rabi oscillations (usually connected with photon antibunching), we have observed fluctuations of the atomic magnetic orientation by measuring photon correlations between orthogonal polarization components.

Using a simple model we have given evidence for the following dynamical processes causing strong circular cross correlations in resonance fluorescence: 
Spontaneous emission of circularly polarized photons causes instantaneous orientation. Subsequent photons are preferentially absorbed and emitted with identical polarization. This memory effect leading to correlated absorption of photons with equal polarization and thus to increased momentum diffusion of an atom with a multi-level structure has been discussed in [17, 20]. In our experiments we can clearly isolate this effect by observation of an anticorrelation of circularly polarized photons successively emitted with opposite handedness.

Subsequent optical pumping induced by atomic motion in the light field causes relaxation of the orientation clearly seen in the photon correlations. In a sense we have seen the elementary sub-Doppler cooling and heating forces in a $\sigma^{+} \sigma^{-}$-molasses at work.

We thank Svenja Knappe for providing some basic techniques in an early stage of the experiment. We are also pleased to acknowledge Frans E. van Dorsselaer and Gerhard Nienhuis for fruitful discussions and sharing their insight into elementary atomic processes. This work is supported by the Deutsche Forschungsgemeinschaft (DFG).

\section{References}

[1] H.J. Kimble, M. Dagenais, and L. Mandel, Photon Antibunching in Resonance Fluorescence, Phys. Rev. Lett. 39, 691 (1977)

[2] F.-M. Rateike, G. Leuchs, and H. Walther, results cited by J.D. Cresser, et al., in Dissipative Systems in Quantum Optics, edited by R. Bonifatio, Topics in Current Physics, vol. 27 (Springer, Berlin, 1982), p. 21.

[3] F. Diedrich and H. Walther, Nonclassical Radiation of a single Stored Ion, Phys. Rev. Lett. 58, 203 (1987).

[4] V. Gomer, F. Strauch, B. Ueberholz, S. Knappe, D. Meschede, Single atom dynamics revealed by photon correlations, Phys. Rev. A58, R1657 (1998).

[5] V. Gomer, F. Strauch, B. Ueberholz, S. Knappe, D. Frese, D. Meschede, Decoding the dynamics of a trapped atom in a MOT, Appl. Phys. B 67, 689-697 (1998)

[6] R.J.Glauber, The Quntum Theory of Optical Coherence, Phys. Rev. 130, 2529 (1963); R.J.Glauber, Coherent and Incoherent States of the Radiation Fields, Phys. Rev. 131, 2766 (1963) Phys. Rev. 131, 2766 (1963)

[7] D.F. Walls and G.J. Milburn, Quantum Optics, Springer Verlag, Berlin Heidelberg New York 1994. 
[8] R. Hanbury Brown and R.Q. Twiss, Correlation between photons in two coherent beams of light, Nature 177, 27 (1956);

[9] R. Loudon, The quantum theory of light, Oxford University Press, second edition, 1983.

[10] E.L. Raab, M. Prentiss, A. Cable, S. Chu, and D.E. Dritchard, Trapping of Neutral Sodium Atoms with Radiation Pressure, Phys. Rev. Lett.59, 2631 (1987); for a recent review see C.G. Townsend, N.H. Edwards, C.J. Cooper, K.P. Zetie, and C.J. Foot, A.M. Steane, P. Szriftgiser, H. Perrin, and D. Dalibard, Phase-space density in the magneto-optical trap, Phys. Rev.A52, 1423 (1995)

[11] H. Schadwinkel, U. Reiter, V. Gomer, and D. Meschede, Magnetooptical trap as an optical lattice, Phys. Rev. A61, 013409 (2000)

[12] A. Rauschenbeutel, H. Schadwinkel, V. Gomer, D. Meschede, Standing light fields for cold atoms with intrinsically stable and variavle time phases, Optics Comm. 148, 45 (1997)

[13] C. Jurczak, B. Desruelle, K. Sengstock, J.-Y. Courtois, C. I. Westbrook, and A. Aspect, Atomic Transport in an Optical Lattice: An Investigation through Polarization-Selective Intensity Correlations, Phys. Rev. Lett. 77, 1727 (1996)

[14] P.B. Coates, The correction for photon "pile-up" in the measurement of radiative lifetimes, J. Sci. Instr. (J. Phys. E), Ser. 2, Vol. 1, 878 (1968)

[15] The difference in time constants for $g_{l r}^{(2)}(\tau)$ and $g^{(2)}(\tau)$ rapidly growing with $F$ has been also observed in quantum Monte-Carlo simulations performed for an atom with $F \rightarrow F+1$ transition in a $1 \mathrm{D}-\sigma^{+} \sigma^{-}$-molasses by F. E. van Dorsselaer and G. Nienhuis (private communication). Similar effect of the growing difference in the cooling and magnetization decay times with increasing angular momentum has been recently mentioned in G. Raithel, W. D. Phillips, and S. L. Rolston, Magnetization and spin-flip dynamics of atoms in optical lattices, Phys. Rev. A 58, R2660, (1998)

[16] W. M. Klipstein, S. K. Lamoreaux, and E. N. Fortson , Observation of Spontaneous Spin Polarization in an Optically Pumped Cesium Vapor, Phys. Rev. Lett. 76, 2266 (1996)

[17] J. Dalibard, C. Cohen-Tannoudjii, Laser cooling below the Doppler limit by polarization gradients: simple theoretical models, J. Opt. Soc. Am. B 6, 2058 (1989) 
[18] C. Cohen-Tannoudji, Atoms motion in laser light, in: J. Dalibard, J.-M. Raimond und J. Zinn-Justin (eds.), Fundamental Systems in Quantum Optics,1, North-Holland, Amsterdam 1992

[19] A. M. Steane and C. J. Foot, Laser cooling below the Doppler limit in a magneto-optical trap, Europhys. Lett. 14(3), 231 (1991)

[20] Y. Castin and K. Mølmer, Atomic momentum diffusion in a $\sigma_{+}-\sigma_{-}$ laser configuration: influence of an internal sublevel structure, J. Phys. B23, 4101 (1990) 